\title{
Dynamic lipopolysaccharide transfer cascade to TLR4/MD2 complex via LBP and CD14
}

\author{
Soo Jin Kim \& Ho Min Kim ${ }^{*}$ \\ Graduate School of Medical Science \& Engineering, KAIST, Daejeon 34141, Korea
}

Toll-like receptor 4 (TLR4) together with MD2, one of the key pattem recognition receptors for a pathogen-associated molecular pattern, activates innate immunity by recognizing lipopolysaccharide (LPS) of Gram-negative bacteria. Although LBP and CD14 catalyze LPS transfer to the TLR4/MD2 complex, the detail mechanisms underlying this dynamic LPS transfer remain elusive. Using negative-stain electron microscopy, we visualized the dynamic intermediate complexes during LPS transfer-LBP/LPS micelles and temary CD14/LBP/ LPS micelle complexes. We also reconstituted the entire cascade of LPS transfer to TLR4/MD2 in a total internal reflection fluorescence (TIRF) microscope for a single molecule fluorescence analysis. These analyses reveal longitudinal LBP binding to the surface of LPS micelles and multi-round binding/unbinding of CD14 to single LBP/LPS micelles via key charged residues on LBP and CD14. Finally, we reveal that a single LPS molecule bound to CD14 is transferred to TLR4/MD2 in a TLR4-dependent manner. These discoveries, which clarify the molecular mechanism of dynamic LPS transfer to TLR4/MD2 via LBP and CD14, provide novel insights into the initiation of innate immune responses. [BMB Reports 2017; 50(2): 55-57]

*Corresponding author. E-mail: hm_kim@kaist.ac.kr

https://doi.org/10.5483/BMBRep.2017.50.2.011

Received 21 January 2017

Keywords: CD14, Innate immunity, LBP, LPS transfer, Single-molecule fluorescence analysis, TLR4/MD2, Transmission electron microscopy

Abbreviations: CD14, cluster of differentiation 14; LBP, LPS-binding protein; LPS, lipopolysaccharide; MD2, myeloid differentiation factor 2; TEM, transmission electron microscopy; TLR4, Toll-like receptor 4

Perspective to: Je-Kyung Ryu and Soo Jin Kim et al. (2017), Reconstruction of LPS transfer cascade reveals structural determinants within LBP, CD14, and TLR4-MD2 for efficient LPS recognition and transfer, Immunity, 46(1), 38-50, doi: 10.1016/j.immuni.2016.11.007.
Pattern-recognition receptors (PRRs), including Toll-like receptors (TLRs) and cytoplasmic receptors (i.e., NOD-like receptors, RIG-I-like receptor, C-type lectin receptors), recognize microbial components and activate the innate immune response for a rapid host defense against pathogen infection. Among 13 members of the TLR family, TLR4 in complex with MD2 senses lipopolysaccharide (LPS), which is the major component of the outer membrane of Gram-negative bacteria. Because LPS forms aggregates or micelles in aqueous solutions due to their hydrophobic multi-acyl chains, transferring one LPS molecule to TLR4/MD2 constitutes one of the critical steps in the recognition of a pathogen-associated molecular pattern (PAMP) of Gram-negative bacteria. The accessory proteins LBP and CD14 mediate the sensitive recognition of LPS as well as their efficient transfer to the TLR4/MD2 complex. Binding of LPS to the TLR4/MD2 complex induces the dimerization of the TLR4/MD2 complex, which brings the cytosolic TIR domains of TLR4 into close enough proximity to recruit downstream adaptor molecules, such as MyD88, TIRAP, TRIF, and TRAM. These TLR4 signaling cascades activate several transcriptional regulators, such as NF-kB, AP-1, and IFN regulatory factors, eventually resulting the expression of genes involved in the host immune response (Tan Y and Kagan JC (2014) Mol Cell 54, 212-223 doi: 10.1016/j.molcel.2014.03.012). However, the over amplified or dysregulated immune responses to LPS can cause severe systemic inflammation leading to tissue damage, organ failure, and death, a condition called sepsis or septic shock.

In the last decade, the crystal structures of the LPS receptors and accessory proteins have been characterized (Fig. 1A) (Kim $J$ et al (2005) J Biol Chem 280, 11347-11351; Kim HM et al (2007) Cell 130, 906-917; Park BS et al (2009) Nature 458, 1191-1195 doi: 10.1038/nature07830; Eckert JK et al (2013) Immunity 39, 647-660 doi: 10.1016/j.immuni.2013.09.005). LBP, which is a soluble class I acute-phase protein, has an elongated structure with a high affinity for LPS. CD14 is present in a GPI-anchored membrane form on the myeloid cell or a soluble form in the serum. The horseshoe-shaped CD14 possesses an $\mathrm{N}$-terminal hydrophobic pocket that provides a putative binding site for amphipathic LPS. LBP has been known to present LPS to CD14 by binding to LPS and, in turn, CD14 either delivers LPS to the surface TLR4/MD2 complex or mediates the LPS-induced endocytosis of TLR4. MD2, which 
(A)
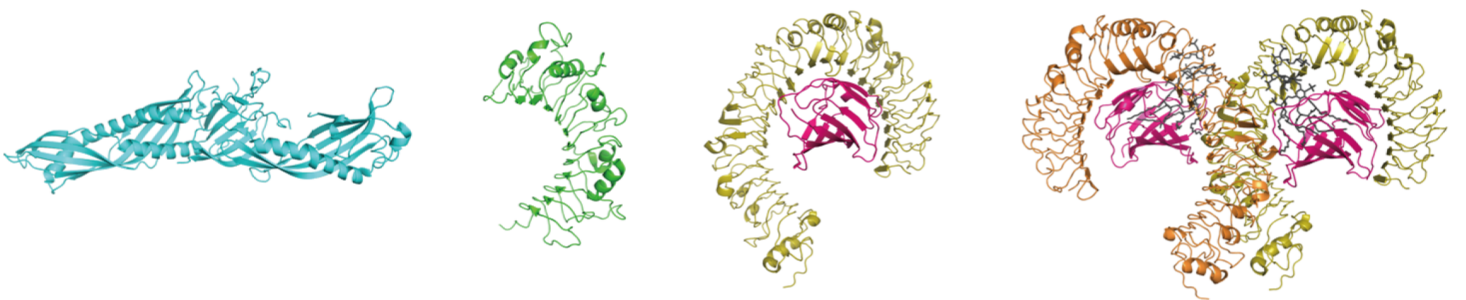

(B)
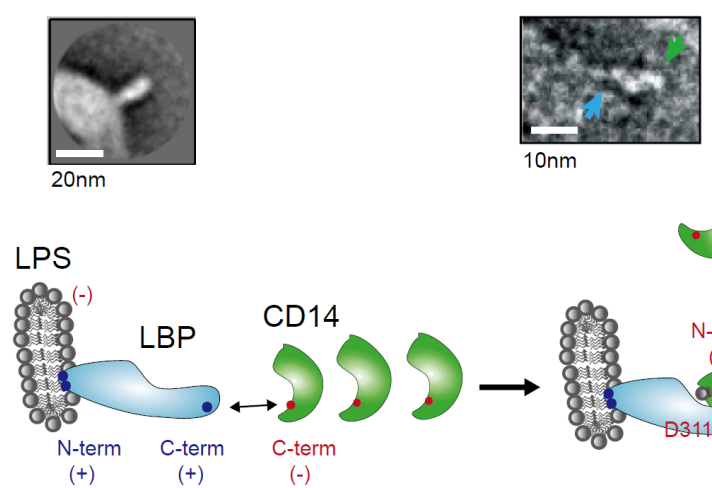
$\begin{aligned} & \text { 1. Longitudal binding of } \\ & \text { 2. Electrostatic interaction } \\ & \text { LBP to LPS micelle }\end{aligned}$ LBP to LPS micelle

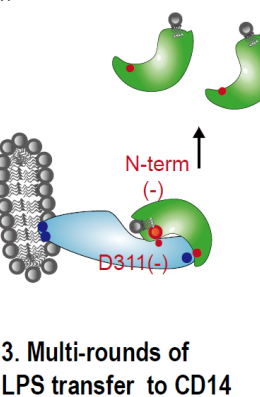

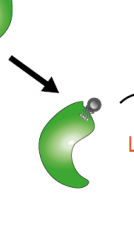

4. TLR4-dependent LPS transfer
TLR4/MD2

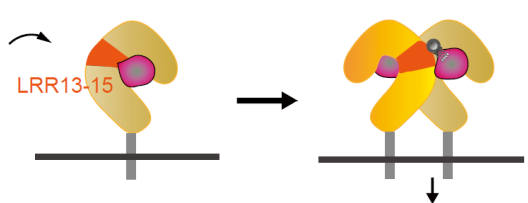

5. LPS/TLR4/MD2 6. TLR4/MD2

dimerization signaling activation

Fig. 1. Lipopolysaccharide transfer cascade to the TLR4/MD2 complex via LBP and CD14. (A) Crystal structure of LBP (PDB: 4M4D), CD14 (PDB: 1WWL), TLR4/MD2 complex (PDB: 2Z64), and TLR4/MD2/LPS complex (PDB: 3FXI). (B) 2D class average of LPS micelle-bound LBP (top left), a representative negative-stained TEM image of LPS micelle/LBP/CD14 (top right, sky-blue arrow: LPS micelle-bound LBP, green arrow: CD14), and schematic model of sequential LPS transport to the TLR4/MD2 complex (bottom). N-terminal tip of LBP binds to LPS micelle and the concave surface in the C-terminal domain of CD14 interacts with C-terminal tip of LBP to obtain a single LPS molecule. The LPS-bound CD14 is rapidly dissociated from the LBP by electrostatic repulsion and another CD14 binds to the LBP. CD14/LPS interacts with LRR13-15 domains of TLR4 to transfer LPS to TLR4/MD2. Two LPS-bound TLR4/MD2 complexes form a M-shaped dimer, followed by activation of the signaling pathway for the innate immune response.

adopts a $\beta$-cup fold structure with a large hydrophobic pocket for LPS binding, forms a stable 1:1 complex with the horseshoe-shaped TLR4. Upon binding of LPS to this pocket, the symmetrical, M-shaped dimer of the TLR4/MD2/LPS complex is formed. Although these structures for LBP, CD14, TLR4/MD2, and the M-shaped TLR4/MD2/LPS dimer have improved our understanding tremendously, they are, by definition, static snapshots of proteins with important dynamic in vivo interactions. The transient nature of the interactions between TLR4/MD2, LBP, CD14 and LPS is advantageous for a rapid immune response but has made it difficult to elucidate the molecular structures of intermediate complexes in the LPS recognition/transfer cascade.

To obtain a clearer picture of these transient intermediates (i.e., LPS-bound LBP, LPS-bound CD14, the LBP/CD14 complex, and the CD14/TLR4/MD2 complex) and to uncover the detailed molecular mechanisms underlying the entire process of LPS transfer, here we used two advanced biophysical techniques - transmission electron microscopy and singlemolecule fluorescence analysis. With negative-stain TEM analysis, we directly visualized dynamic intermediate complexes comprising LBP/LPS micelles, revealing that LBP binds longitudinally to the surface of LPS micelles via its $\mathrm{N}$-terminal basic tip, reminiscent of the cholesteryl ester transfer protein (CETP)/lipoprotein interaction (Zhang L et al (2012) Nat Chem Biol 8, 342-349 doi: 10.1038/nchembio.796). In addition, we reconstituted the entire LPS transfer process from LPS micelles to TLR4/MD2 in a total internal reflection fluorescence (TIRF) microscope. This in vitro reconstitution allowed us to quantitatively monitor the dynamic interactions occurring during this LPS transfer and to dissect the intermediates of each step in the LPS transfer cascade with single molecule or event resolution. We identified that the interaction between LBP and CD14 is transient, lasting only hundreds of milliseconds, and mediated by electrostatic interaction between a basic patch (K319, R322) in the C-terminal domain of LBP and an acidic patch (D251, D297) on the concave surface of the CD14 C-termini. These transient interactions only occur in the presence of LPS and are essential for LPS transfer. We also revealed that CD14 rapidly dissociates from the LBP/LPS complex after receiving one LPS molecule. After the rapid dissociation of CD14/LPS, which was mediated by 
electrostatic repulsion between LBP D311 and CD14 $\mathrm{N}$-termini, LBP retains its binding to LPS micelles and recruits another CD14. Meanwhile, CD14 delivers a single LPS molecule to MD2 in a TLR4-dependent manner, particularly the LRR13-LRR15 domain in TLR4 (Fig. 1B).

In conclusion, our integrative investigation of the dynamic intermediates that form during the entire LPS transfer process revealed an unprecedented level of detail into how LPS molecules are recognized and transferred to initiate the innate immune response. Not only that, these provide a blueprint for future therapeutic strategies by modulating LPS transfer to TLR4/MD2.

\section{ACKNOWLEDGEMENTS}

This work was supported by the Korean Health Technology R\&D Project, Ministry of Health and Welfare (HI15C0508), and the Intelligent Synthetic Biology Center of Global Frontier Project from the Ministry of Science, ICT, and Future Planning. 